\title{
11.
}

\section{Über die Erzeugung der Curven dritter Ordnung durch gerade Linien, und über geometrische Definitionen dieser Curven.}

(Von Herrn H. Gra/smann, Oberlehrer an der Friedrich-Wilhelm-Schule zu Stettin.)

In einem Aufsatze über Curven dritter Ordnung, im 34ten Bande dieses Journals, behauptet Herr Professor Plücker, es gebe noch keine allgemeine geometrische Definition einer Curve dritter Ordnung, und schliefst daraus, dafs eine rein geomeirische Behandlung dieser Curven, und also um so mehr der höheren Curven, gegenwärtig noch unmöglich sei. Nun habe ich im 31 ten Bande dieses Journals die Grundzüge einer rein geometrischen Behandlung der höheren Curven zu geben versucht, und habe dort, namentlich für die Curven dritter Ordnung, eine geometrische Definition aufgestellt, deren Allgemeinheit ich dort nachgewiesen habe (S.15 bis 17); ich könnte daher den Gegenstand als abgemacht ansehen, und mich damit beruhigen, dafs Herrn Plücker jener Band des Journals nicht zu Gesichte gekommen sei, wenn ich nicht befürchten müfste, dafs durch die so entschieden ausgesprochene Behauptung mancher Leser irre geführt werden möchte. Ich werde daher den Gegenstand hier noch einmal, und zwar von einem umfassenderen Gesichtspuncte aus aufnehmen.

Die einfachsten geometrischen Definitionen der Curven dritter Ordnung, deren jede diese Curven in ihrer ganzen Allgemeinheit darstellt, würden folgende drei sein; zwischen denen man, um eine methodische Behandlung darauf zu gründen, wählen kann:

No. 1. Der geometrische Ort der gemeinschaftlichen Spitze zweier Dreiecke, deren Winkel an der Spitze einen gemeinschaftlichen Schenkel haben, während von den beiden nicht gemeinschaftlichen Schenkeln derselben jeder durch einen gegebenen Punct geht und von den 4 Endpuncten der Grundseiten jeder in einer gegebenen geraden Linie liegt, ist eine Curve dritter Ordnung.

No. 2. Wenn die Seiten eines veränderlichen Vierecks und eine Diagonale desselben um feste Puncte sich drehen, und die von der Diagonale nicht getroffenen Ecken in festen Geraden liegen, so ist der geometrische Crelle's Journal f. d. M. Bd. XXXVI. Heft 2. 
Ort jeder von der Diagonale getroffenen Ecke des Vierecks eine Curve dritter Ordnung.

No. 3. Der geometrische Ort eines Punctes, dessen Verbindungslinien mit 3 gegebenen Puncten 3 gegebene Gerade so schneiden, dafs die 3 Durchschnittspuncte in gerader Linie liegen, ist eine Cnrve dritter Ordnung *).

Von diesen 3 Definitionen habe ich die erste in dem oben angeführten Aufsatze als eine alle Curven dritter Ordnung umfassende nachgewiesen, und ich habe dem Beweise nichts weiter hinzuzufügen. Dafs der geometrische Ort, welcher in der zweiten und dritten Definition genannt ist, gleichfalls eine Curve dritter Ordnung sei, ist dort ebenfalls bewiesen. Es bleibt nur übrig, zu zeigen, dafs auch jede dieser beiden letzten Definitionen alle Curven dritter Ordnung umfafst. Für die zweite Definition will ich hier den Nachweis vollständig liefern, während ich für die dritte nur den Gang des Beweises angeben werde.

Es sei $x u y u_{1}$ (Taf. I. Fig. 1.) das veränderliche Viereck, dessen Seiten $x u, u y, y u_{1}, u_{1} x$ und dessen Diagonale $x y$ sich beziehlich um die festen Puncte $a, b, a_{1}, b_{1}$ und $d$ drehen und dessen Ecken $u$ und $u_{1}$ sich beziehlich in den festen Geraden $C$ und $C_{1}$ bewegen. Der Kürze wegen bezeichne ich, wie im ersten Aufsatze, die Verbindungslinie zweier verschiedener Puncte $a$ und $b$ durch $a b$, und den Durchschnitt zweier verschiedener Geraden $\boldsymbol{A}$ und $\boldsymbol{B}$ durch $\boldsymbol{A B}$; wenn mehrere solche Ausdrücke ohne Klammern nebeneinander geschrieben sind, so soll die Construction in der Ordnung fortschreiten, wie diese Ausdrücke von links nach rechts hin folgen. Dann läfst sich nachweisen, dafs die von dem Puncte $x$ construirte Curve dritter Ordnung die 9 Puncte

$$
a, a_{1}, d, C C_{1},(a b)\left(a_{1} b_{1}\right), \quad d b C, d b_{1} C_{1}, a b C_{1}, a_{1} b_{1} C
$$

enthält, die ich nach der Reihe beziehlich mit

$$
a, a_{1}, d, e, \quad f, \quad g, \quad y_{1}, h, \quad h_{1}
$$

bezeichnen will. In der That: liegt $x$ in einem der Puncte $a, a_{1}$, oder $d$, so kann die Verbindungslinie zwischen $x$ und diesem Puncte jede Richtung

*) Ich verstehe hier unter Curve dritter Ordnung (algebraisch gefafst) die Gesanmtheit der Puncte, deren Coordinaten einer algebraischen Gleichung genügen, welche in Bezug auf diese Coordinaten vom 3ten Grade ist; und zwar auch dann noch, wenn beliebig viele der Constanten Null werden. Ich würde hiefür den Ausdruck Punctyebilde 3ten Grades vorziehen, wenn ich nicht befürchtete, dadurch undeutlicher zu werden. 
annehmen, und es läfst sich daher dann leicht ein Viereck von der verlangten Art zeichnen. Liegt z. B. $x$ in $a$, so giebt $x a_{1} C_{1}$ den Punct $u_{1},\left(u_{1} b_{1}\right)(x d)$ den Punct $y, y b C$ den Punct $u$; und verbindet man nun noch $u$ mit $a$, so hat das so construirte Viereck $x u_{1} y u$ die verlangte Eigenschaft, d. h. $a$ ist ein Punct der von $x$ construirten Curve. Liegt $x$ nicht in einem dieser 3 Puncte, so haben die 3 von $x$ ausgehenden Geraden $x a, x d, x a_{1}$ bestimmte Richtungen, und es werden alle diejenigen Puncte $x$-Puncte der Curve sein, für welche die 3 Geraden

$$
x a C b, \quad x a_{1} C_{1} b_{1}, \quad x d
$$

durch denselben Punct $(y)$ gehen. Liegt nun $x$ in $C$ oder in $a b$, so wird die erste jener 3 Geraden gleich $x b$; liegt $x$ in $C_{1}$ oder in $a_{1} b_{1}$, so wird die zweite jener Geraden gleich $x b_{1}$. Liegt also $x$ in dem Durchschnitt von $C$ und $C_{1}$, oder von $a b$ und $a_{1} b_{1}$, oder von $C$ und $a_{1} b_{1}$, oder von $C_{1}$ und $a b$, so gehen jene 3 Geraden durch denselben Punct $x$; d. h. es sind diese Durchschnitte Puncte der von $x$ construirten Curve, d. h. es liegen $e, f, h, h_{1}$ in dieser Curve. Endlich: liegt $x$ im Durchschnitt von $d b$ und $C$, so wird die erste jener 3 Geraden gleich $x b$ und die dritte gleich $x d$; was mit $x b$ zusammenfällt: also gehen dann wieder die 3 Geraden durch denselben Punct; d. h. g ist ein Punct der Curve, und aus demselben Grunde auch $y_{1}$. Es sind daher alle 9 oben genannten Puncte als Puncte der durch die Ecke $x$ beschriebenen Curve dritter Ordnung nachgewiesen.

Nach diesen Vorbereitungen hat es nun keine Schwierigkeit mehr, die in No. 2. angegebene Erzeugung als eine allgemeine, d. h. als eine solche nachzuweisen, durch welche jede beliebige Curve dritter Ordnung erzeugt werden kann. In der That: ist irgend eine Curve dritter Ordnung gegeben, so schreibe man ihr irgend ein Viereck fheh ein, dessen Seiten $f h$, he, $e h_{1}, h_{1} f$ die Curve zum dritten Male, beziehlich in den Puncten $a, g_{1}, g, a_{1}$ treffen mögen und ziehe von irgend einem andern Puncte $d$ der Curve, der aber nicht in dem Durchschnitt der beiden Linien ag und $a_{1} g_{1}$ liegt, nach zwei auf einander folgenden der letztgenannten Puncte, z. B. nach $g_{1}$ und $g$, die Geraden, welche die gegenüberstehenden Seiten des Vierecks beziehlich in $b_{1}$ und $b$ schneiden mögen; dann sind die 9 so gewonnenen Puncte der Curve zufolge der vorher gegebenen Entwickelung zugleich Puncte derjenigen Curve dritter Ordnung, welche durch eine Ecke $x$ eines Vierecks $x u y u_{1}$ beschrieben wird, dessen Ecken $u$ und $\boldsymbol{u}_{\mathbf{1}}$ sich beziehlich in den Geraden $e h_{1}$ und $e \boldsymbol{h}$ bewegen, während die Seiten $x u, u y, y u_{1}, u_{1} x$ und die Diagonale $x y$ be- 
ziehlich um die Puncte $a, b, b_{1}, a_{1}, d$ sich drehen. Diese so erzeugte Curve hat mit der gegebenen 9 Puncte gemein; und zwar, da $d$ nicht in dem durch die übrigen 8 Puncte schon bedingten Puncte (nämlich in dem Durchschnittspuncte der Geraden $a g$ und $a_{1} g_{1}$ ) liegt, 9 solche Puncte, durch welche eine Curve dritter Ordnung bestimmt ist. Somit fällt die durch die Ecke $x$ erzeugte Curve mit der gegebenen zusammen, und die Definition No. 2. ist als allgemein nachgewiesen. Hiermit ist zugleich gelegentlich der nachstehende Satz bewiesen:

Wenn man einer Curve dritter Ordnung ein Viereck (fhe $\left.h_{1}\right)$ einschreibt, dessen 4 Seiten $\left(f l h, h e, e h_{1}, h_{1} f\right)$ die Curve beziehlich in 4 neuen Puncten $\left(a, g_{1}, g, a_{1}\right)$ treffen, und man zieht von zweien dieser letztgenannten 4 Puncte, die in gegenüberliegenden Seiten jenes Vierecks liegen (z. B. von $g$ und $a$, oder von $g_{1}$ und $a_{1}$ ), die Geraden beziehlich nach einem 9ten und einem 10ten Puncte der Curve ( $d$ und $x$ ), was auf 4 Arten möglich ist: so geht jedesmal die Verbindungslinie derjenigen Puncte ( $b$ und $u$, oder $b_{1}$ und $u_{1}$ ), worin diese Geraden beziehlich die gegenüberliegenden Seiten des Vierecks treffen, durch einen und denselben Punct $(y)$ der Verbindungslinie des 9ten und 10ten Puncts; welche jener 4 möglichen Arten der Verbindung man auch wählen mag.

Den Beweis davon, dafs auch die dritte Definition allgemein sei, will ich hier nur mehr andeuten, als ausführen. Es sei $x$ (Fig. 2.) der veränderliche Punct, dessen Verbindungslinien mit den festen Puncten $\boldsymbol{a}, \boldsymbol{b}, \boldsymbol{c}$ beziehlich die festen Geraden $\boldsymbol{A}, \boldsymbol{B}, \boldsymbol{C}$ so schneiden, dafs die drei Durchschnittspuncte $\boldsymbol{u}, \boldsymbol{v}, \boldsymbol{w}$ in gerader Linie liegen. In dem angeführten Aufsatze (S. 17) habe ich gezeigt, dafs dann der geometrische Ort von $x$ eine Curve dritter Ordnung ist, welche durch folgende 9 Puncte geht:

$$
a, b, c, B C, C A, A B, b c A, c a B, a b C,
$$

die ich beziehlich mit

$$
a, \quad b, \quad c, \quad a_{1}, \quad b_{1}, \quad c_{1}, \quad \alpha, \quad \beta, \quad \gamma
$$

bezeichnen will. Nun läfst sich leicht zeigen, dafs man jeder Curve dritter Ordnung Dreiecke einschreiben kann, deren entsprechende Seiten sich auf der Curve schneiden. Es seien $a b c$ und $a_{1} b_{1} c_{1}$ zwei solche, einer gegebenen Curve dritter Ordnung eingeschriebene Dreiecke, deren entsprechende Seiten sich beziehlich in den Curvenpuncten $\gamma, \alpha, \beta$ schneiden. Dann sind diese 9 Puncte zugleich Puncte der Curve dritter Ordnung, welche durch einen Punct $x$ beschrieben wird, dessen Verbindungslinien mit $\boldsymbol{a}, \boldsymbol{b}, \boldsymbol{c}$ die Geraden 
$b_{1} c_{1}, c_{1} a_{1}, a_{1} b_{1}$ beziehlich in dreien in gerader Linie liegenden Puncten schneiden; und zwar sind es 9 solche Puncte, durch welche die Curve dritter Ordnung bestimmt ist; folglich fällt die durch $x$ erzeugte Curve mit der gegebenen zusammen, und die Definition No. 2. ist als allgemein nachgewiesen.

Um den Gegenstand endlich noch von einem allgemeineren Gesichtspuncte aus zu betrachten, werde ich den allgemeinen Satz über die Art, wie Curven dritter Ordnung und Curven dritter Classe durch Bewegung von geraden Linien erzeugt werden können, aufstellen; aus welchem man dann beliebig viele rein geometrische Definitionen der Curven dritten Grades ableiten kann.

Um diesen Satz in leicht fafslicher Form aussprechen zu können, will ich mich des Begriffs der offenen (nicht geschlossenen) Figur bedienen. Die offene Figur besteht aus einer Reihe von Puncten und geraden Linien, in der Art, dafs auf jeden Punct eine durch ihn gehende Gerade, und auf jede Gerade ein in ihr liegender Punct folgt, bis endlich die Reihe entweder mit einem Puncte oder einer Geraden schliefst; wie sie denn auch entweder mit einem Puncte oder mit einer Geraden beginnt. Punct und Gerade will ich zusammen Elemente nennen; das Element, mit welchem jene Reihe beginnt, will ich Anfangs-Element; das, womit sie schliefst, End -Element, beide zusammen Grenz-Elemente der offenen Figur nennen; alle Puncte der Reihe, die nicht Grenz-Elemente sind, nenne ich Ecken, alle Geraden der Reihe, die nicht Grenz - Elemente sind, Seitenlinien, oder kurzweg Seiten der offenen Figur. Es sind hiernach also 3 Fälle möglich: entweder beide Grenz-Elemente sind Puncte; dann hat die Figur eine Seite mehr als sie Ecken hat; oder beide Grenz-Elemente sind Gerade; dann hat sie eine Ecke mehr, als sie Seiten hat; oder endlich: Ein Grenz-Element ist ein Punct, das andere eine Gerade; dann hat sie eben so viele Ecken als Seiten und verwandelt sich, wenn der Grenzpunct in der Grenzlinie liegt, in eine geschlossene Figur. Wenn die offene Figur sich stetig verändert, so können bei dieser Veränderung in besonderen Übergangsfällen 2 aufeinander folgende Gerade der Reihe, oder 2 aufeinander folgende Puncte derselben zusammenfallen; alsdann kann man dort jeden Punct der zusammenfallenden Geraden als zwischenliegende Ecke, hier jede durch die zusammenfallenden Puncte gelegte Gerade als zwischenliegende Seitenlinie der offenen Figur auffassen. Der Satz von Erzeugung der Curven dritten Grades wird sich nun in folgender Gestalt darstellen lassen:

Wenn in einem Verein drcier offener Figuren, deren Anfangs-Elemente und deren End-Elemente zusammenfallen, alle Seiten derselben um 
feste Puncte und alle Ecken derselben in festen Geraden sich bewegen, so beschreibt jedes Grenz-Element ein Gebilde dritten Grades ${ }^{*}$ ); und aufser dieser giebt es keine durch blofse gerade Linien bedingte Erzeugung der Gebilde dritten Grades.

Der Beweis des ersten Theils dieses Satzes ist in dem oben angeführten Aufsatze gegeben, in welchem der Satz für höhere Curven aufgestellt ist. Dafs es aufserdem keine andere lineale Erzeugungsweise der Gebilde dritten Grades giebt, folgt leicht aus demselben allgemeinen Satze für höhere Curven, indem sich leicht zeigen läfst, dafs alle andern Erzeugungs-Arten entweder höhere oder niederere Gebilde liefern. Der Satz, den ich hier aufgestellt habe, bietet 3 wesentlich verschiedene Fälle dar, nämlich: erstens, wenn die 3 offenen Figuren Puncte zu Grenz-Elementen haben, so beschreiben beide Puncte, jeder eine Curve dritter Ordnung; oder zweitens, wenn die GrenzElemente gerade Linien sind, dann umhüllen diese Geraden jede eine Curve dritter Clạsse; oder endlich, wenn von den Grenz-Elementen eins ein Punct, das andere eine Gerade ist, so wird von jenem eine Curve dritter Ordnung beschrieben, von dieser eine Curve dritter Classe umhültt. Ich will hierbei noch bemerken, dafs von den 3 oben zu einer Definition aufgestellten Erzeugungs-Arten No. 2. zu dem ersten dieser 3 Fälle, und No. 1. und No. 3. zu dem letzten derselben gehören.

Stettin, den 28ten November 1847.

*) Ich sage, ein Punct beschreibe ein Gebilde nten Grades, wenn er eine Curve nter Ordnung (ein Punctgebilde nten Grades) durchläuft, und eine Gerade beschreibe ein Gebilde nten Grades, wenn sie eine Curve nter Classe (ein Liniengebilde nten Grades) umhüllt. 